
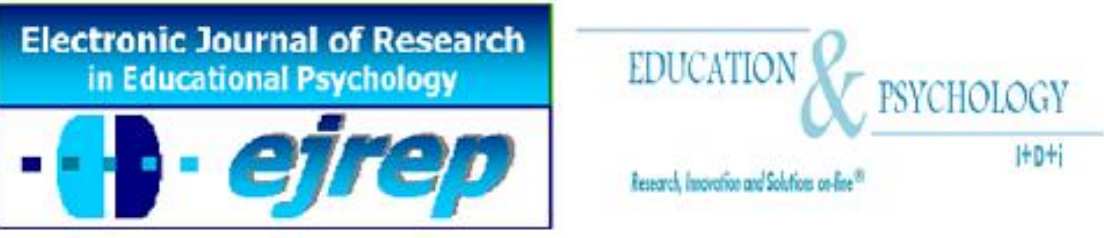

\title{
Relación entre el Rendimiento Escolar y la Sintomatología Depresiva en Niños Españoles
}

\section{Mireia Orgilés ${ }^{1}$, Marta Gómez ${ }^{1}$, José A. Piqueras ${ }^{1}$ y José P. Espada}

${ }^{1}$ Departamento de Psicología de la Salud, Universidad Miguel Hernández, Elche (Alicante)

\section{España}

Correspondencia: Mireia Orgilés. Universidad Miguel Hernández. Edificio Altamira. Avda. de la Universidad s/n. Elche, 03202, Alicante. España. E-mail: morgiles@umh.es

(C) Education \& Psychology I+D+i and and Ilustre Colegio Oficial de Psicólogos de Andalucía Oriental (Spain) 


\section{Resumen}

Introducción. A pesar de los datos que evidencian la relación entre la depresión y la disminución del rendimiento escolar, se carece de estudios llevados a cabo con población infantil española. El objetivo del presente trabajo es examinar el rendimiento escolar de los niños españoles en relación con la depresión infantil y el sexo.

Método. Participaron 658 niños españoles de edades comprendidas entre 8 y 12 años, el $49.6 \%$ varones, que completaron el Inventario de Depresión Infantil e informaron de sus calificaciones (número de suspensos y número de sobresalientes) y del número de veces que habían repetido curso.

Resultados. Los resultados del Análisis Multivariado de la Varianza muestran que los niños con sintomatología depresiva tienen un peor rendimiento escolar (más suspensos, menos sobresalientes y más cursos repetidos) que los que no presentan sintomatología. Únicamente se hallaron diferencias en función del sexo en el número de cursos repetidos, mayor en las niñas que en los niños. En ninguna de las variables estudiadas se encontraron diferencias en la interacción entre la sintomatología depresiva y el sexo.

Discusión y conclusión. Es conveniente la detección temprana de sintomatología depresiva en el ámbito escolar con el fin de prevenir problemas de rendimiento académico.

Palabras Clave: sintomatología depresiva, rendimiento escolar, estudio descriptivo, población infantil. 


\title{
Relations between School Performance and Depression Symptomatology in Spanish Children.
}

\begin{abstract}
Introduction. Despite data showing the relationship between depression and decreased school performance, there is a lack of studies with Spanish children. The objective of this research is to examine school performance in function of depression and gender.

Method. Participants were 658 Spanish children aged between 8 and 12 years, $49.6 \%$ male, who completed the Child Depression Inventory and reported their scores (the number of courses evaluated as Excellent and Failure) and the number of grades they had repeated.

Results. The results of Multivariate Analysis of Variance show that children with depressive symptoms have poorer school performance (more courses evaluated as Failure, less courses evaluated as Excellent, and more repeated grades) than those without symptoms. Differences by gender were only found in the number of repeated grades, higher in girls than in boys. None of the variables studied showed differences in the interaction of depressive symptoms and gender.
\end{abstract}

Discussion and Conclusion. It is necessary the early detection of depressive symptoms at schools in order to prevent problems at academic performance.

Keywords: depressive symptoms, school performance, descriptive study, childhood. 


\section{Introducción}

La prevalencia de la depresión infantil en España se sitúa entre el 5 y el 10\% (Del Barrio, 2007), pero asciende al 14\% en población clínica (López, Alcántara, Fernández, Castro y López, 2010). La sintomatología asociada a la depresión infantil es variada y difiere de la que presentan los adultos. Según Del Barrio (2007) entre los síntomas más característicos de la depresión en los niños se encuentran los problemas de conducta, la irritabilidad, las rabietas y la desobediencia. Además, de forma similar a los adultos, los niños presentan baja autoestima, cambios en el sueño, pérdida o aumento del apetito y del peso, aislamiento social, hiperactividad, disforia e ideación suicida. La sintomatología depresiva infantil está relacionada con dificultades a nivel psicosocial con respecto a la familia, la escuela y las relaciones con los iguales (Levendosky, Okun y Parker, 1995). A partir de una revisión de estudios sobre depresión infantil, Del Barrio (1997) clasificó los síntomas en función de si el área afectada era la emocional, motora, cognitiva, social, conductual o psicosomática, incluyendo dentro del área cognitiva la falta de concentración y el descenso del rendimiento académico.

Desde los primeros estudios realizados la depresión infantil se ha asociado con frecuencia a la disminución del rendimiento escolar (Blechman, Mcenroe, Carella y Audette 1986; Doménech y Polaino-Lorente, 1990; Feshbach y Feshbach, 1987; Kaslow, Rehm y Siegel, 1984; Nissen, 1989; Shafii y Shafii, 1995). En una de las primeras investigaciones llevadas a cabo, Bauersfeld (1972) concluyó que el 13\% de los niños con fracaso escolar manifestaba depresión. En estudios posteriores se ha observado también una mayor incidencia de depresión en los niños que han repetido curso que en los que nunca lo habían hecho (Doménech y Polaino-Lorente, 1990). Además del número de repeticiones de curso, según Cabrera y Jiménez (1990) se evidencia un mayor número de suspensos en distintas asignaturas en niños con problemas del estado de ánimo.

Durante los últimos años el estudio de la relación entre la depresión infantil y el rendimiento escolar ha suscitado gran interés. En una investigación llevada a cabo por Fletcher (2008) se concluye que las adolescentes con depresión tienen una probabilidad menor de graduarse en el instituto y muestran una disminución en su asistencia a la escuela. La Oficina Regional Europea de la Organización Mundial de la Salud (Suhrcke y Nieves, 2011), en un estudio sobre el impacto de la salud y de las conductas saludables en el rendimiento escolar, 
confirma la influencia negativa que la ansiedad y la depresión ejercen sobre los resultados académicos. La dificultad de concentración, síntoma habitual en los niños con sintomatología depresiva, parece determinar los problemas para obtener buenas notas (Cole, 1991; Patterson y Stoolmiller, 1991; Roeser, van der Wolf y Strobel, 2001). En un reciente trabajo de Bernaras, Jaureguizar, Soroa, Ibabe y de las Cuevas (2013) se halló una correlación inversa y significativa entre la sintomatología depresiva y el rendimiento académico. Los problemas en las relaciones sociales, en autoconcepto y en desajuste (clínico, personal y escolar) fueron mayores en el grupo de estudiantes que presentaba un diagnóstico de depresión que en aquéllos en los que éste era negativo.

\section{Objetivos}

A pesar de los datos que evidencian la relación entre la depresión y la disminución del rendimiento escolar, son escasos los estudios llevados a cabo con población infantil española. Por ello, el objetivo de este estudio es examinar si existen diferencias en el rendimiento académico en función de presentar o no sintomatología depresiva y teniendo en cuenta el sexo y la edad de los participantes. A partir de la literatura previa (p.e., Fletcher, 2008) se parte de la hipótesis de que los niños con síntomas depresivos presentarán un rendimiento escolar peor (mayor número de suspensos y cursos repetidos y menos sobresalientes) que los que no presentan sintomatología depresiva. De acuerdo a los datos obtenidos por estudios previos en distintos niveles educativos (Echavarri, Godoy y Olaz, 2007; Plazas, Aponte y López, 2006; Requena, 1998; Sarmiento, Vargas y Díaz, 2012) se espera encontrar que las niñas tengan mejor rendimiento académico que los niños.

\section{Método}

\section{Participantes}

Participaron 658 niños de edades comprendidas entre 8 y 12 años $(M=10.85 ; D . T$. $=$ 0.97), el $49.6 \%$ de sexo masculino. El $86.6 \%$ de los participantes eran españoles y el resto eran nacidos en otros países, pero todos ellos de habla hispana. El nivel socioeconómico, determinado por la situación laboral de los padres y la ubicación del colegio en el que estaban escolarizados, era medio. Respecto a su situación familiar, el 82\% de los niños tenían padres casados, el $16 \%$ padres separados o divorciados y el $2 \%$ tenían madre soltera o eran huérfanos. La mayoría de los niños tenían un solo hermano (61\%), el 16\% tenían dos hermanos, el 13.4\% eran hijos únicos, y el resto tenían tres o más hermanos. 
Con el objetivo de comparar el rendimiento académico en función de la sintomatología depresiva, se establecieron dos subgrupos a partir de las puntuaciones de los sujetos en el Inventario de Depresión Infantil (CDI; Kovacs, 1992) utilizándose la puntuación 19 como punto de corte indicativo de depresión (Domènech y Polaino-Lorente, 1990; Ezpeleta, Osa, Gratacos y Pons, 1992). En la Tabla 1 se presentan las características sociodemográficas de los niños con y sin síntomas de depresión. La edad media de los participantes con síntomas depresivos era de $10.77(D . T .=1.031)$ y la de los sujetos que no presentaban síntomas de $10.86(D . T .=$ 0.96). El porcentaje de varones era de $50.2 \%$ y $43.4 \%$ respectivamente en ambos grupos. No se hallaron diferencias significativas entre ambos subgrupos en ninguna de las variables sociodemográficas examinadas.

Tabla 1. Características de la muestra

\begin{tabular}{lcc}
\hline Variables & $\begin{array}{c}\text { Niños con sintomatología } \\
\text { depresiva } \\
(n=50)\end{array}$ & $\begin{array}{c}\text { Niños sin sintomatología } \\
\text { depresiva } \\
(n=608)\end{array}$ \\
\hline Edad & $10.77(1.031)$ & $10.86(0.96)$ \\
Número de hermanos & $1.47(1.030)$ & $1.29(1.13)$ \\
Niños varones $(\%)$ & 50.2 & 43.4 \\
Origen español $(\%)$ & 87.2 & 79.2 \\
Estudios padre $(\%)$ & & 3.8 \\
$\quad$ Universitarios & 5.6 & 3.8 \\
Medios & 13.2 & 82.7 \\
Básicos & 74.9 & 9.7 \\
No se sabe & 16.3 & \\
Estudios madre $(\%)$ & & 4.2 \\
Universitarios & 3.6 & 90.4 \\
Medios & 10.9 & 60.4 \\
Básicos & 59.5 & 23 \\
No se sabe & 26 & 93.7 \\
Padre trabaja actualmente $(\%)$ & & \\
Madre trabaja actualmente $(\%)$ & 93.9 & \\
\hline
\end{tabular}




\section{Instrumentos}

Los participantes completaron un breve cuestionario sociodemográfico proporcionando información sobre su edad, sexo, trabajo de su madre y su padre, situación familiar, número de hermanos y país de nacimiento. Además, completaron el Inventario de Depresión Infantil (Kovacs, 1992) que evalúa sintomatología depresiva y que se aplica a niños y adolescentes de 7 a 15 años. Está compuesto por 27 ítems con tres opciones de respuesta $(0=$ ausencia de sintomatología; 1 = sintomatología leve; 2 = sintomatología depresiva). Incluye dos subescalas, disforia y autoestima negativa, y una escala total de depresión. La puntuación total del cuestionario puede oscilar entre 0 y 54 . El inventario puede aplicarse de forma individual, lo que se recomienda para niños pequeños y población clínica, o de forma colectiva para población no clínica con fines de investigación o como medida de screening. La versión en español del CDI (Del Barrio y Carrasco, 2004) cuenta con unas propiedades psicométricas satisfactorias, con una consistencia interna de .79. Su fiabilidad test-retest en un intervalo de dos semanas es más baja (alfa $=.38$ ), lo cual es habitual en muestras no clínicas (Del Barrio y Carrasco, 2004).

El rendimiento académico de los participantes se examinó obteniendo información sobre: (a) el número de suspensos en la última evaluación, es decir, el número de asignaturas en las que el alumno obtuvo una puntuación menor a 5 no superando los objetivos del nivel; (b) el número de sobresalientes, es decir, el número de asignaturas con una puntuación de 9 o más puntos indicativos de excelencia en el logro de objetivos del nivel; y (c) el número de veces que el niño había repetido curso.

\section{Procedimiento}

El reclutamiento de los participantes se llevó a cabo en siete colegios públicos y concertados de la provincia de Alicante (España), seleccionados al azar de zonas urbanas y rurales, de la costa y del interior. Se solicitó autorización a los directores de los centros y se obtuvo el consentimiento informado de los padres. El 95\% de los padres dieron la conformidad para que sus hijos participaran en el estudio. Los participantes completaron los cuestionarios de forma anónima en las aulas. Se distribuyeron los cuestionarios y se leyeron en voz alta las instrucciones. Se pidió a los participantes que contestaran con sinceridad y que levantaran la mano si les surgía alguna duda. Ningún participante dejó más del 20\% de los ítems sin responder, de modo que no se excluyó ningún cuestionario del análisis de datos. El Comité de Ética de la institución de la que forman parte los autores aprobó previamente el estudio. 


\section{Análisis Estadístico}

Se llevó a cabo un Análisis Multivariado de la Varianza (MANOVA) incluyendo la variable "presencia o ausencia de sintomatología depresiva" y el sexo como factores fijos; las variables que medían el rendimiento académico (número de suspensos, número de sobresalientes y número de veces que ha repetido curso) se consideraron como variables dependientes, y se ajustó el análisis por la edad. Se halló el tamaño del efecto de Cohen de las comparaciones que resultaron significativas, considerándose 0.20 como un tamaño pequeño, 0.50 medio y 0.80 grande. Todos los análisis estadísticos se llevaron a cabo con el programa PASW.

\section{Resultados}

Atendiendo al punto de corte en el CDI el 7.6\% $(n=50)$ del total de la muestra $(n=$ 658) manifiesta sintomatología depresiva. Los resultados del MANOVA muestran diferencias significativas en todas las variables dependientes en función de tener o no síntomas depresi$\operatorname{vos}(F(1,656)=16.561 ; p<.001)$ y del sexo de los participantes $(F(1,656)=3.658 ; p<$ $.05)$, pero no de la interacción entre ambos factores $(F(3,655)=1.527 ; p>.05)$. Atendiendo a la presencia o ausencia de sintomatología depresiva las diferencias fueron significativas en todas las medidas del rendimiento académico, con un mayor número de repeticiones de curso $(p<.01)$, más suspensos $(p<.001)$ y menos sobresalientes $(p<.001)$ en los niños con síntomas depresivos. El tamaño del efecto fue medio para el número de veces que repiten curso ( $d$ $=0.41)$ y grande para el número de suspensos y sobresalientes $(d=0.71, d=-0.84$, respectivamente). En función del sexo, las diferencias fueron significativas en el número de veces que repiten curso, mayor en las niñas que en los niños $(p<.05)$, con un tamaño del efecto pequeño $(d=-0,38)$. En la Tabla 2 se presentan las medias y desviaciones típicas en las variables de rendimiento académico en los niños con y sin sintomatología depresiva, así como el resultado de las comparaciones entre ambos grupos. 
Tabla 2. Diferencias en el rendimiento académico de los niños en función del sexo y de presentar o no sintomatología depresiva y ajustado por la edad

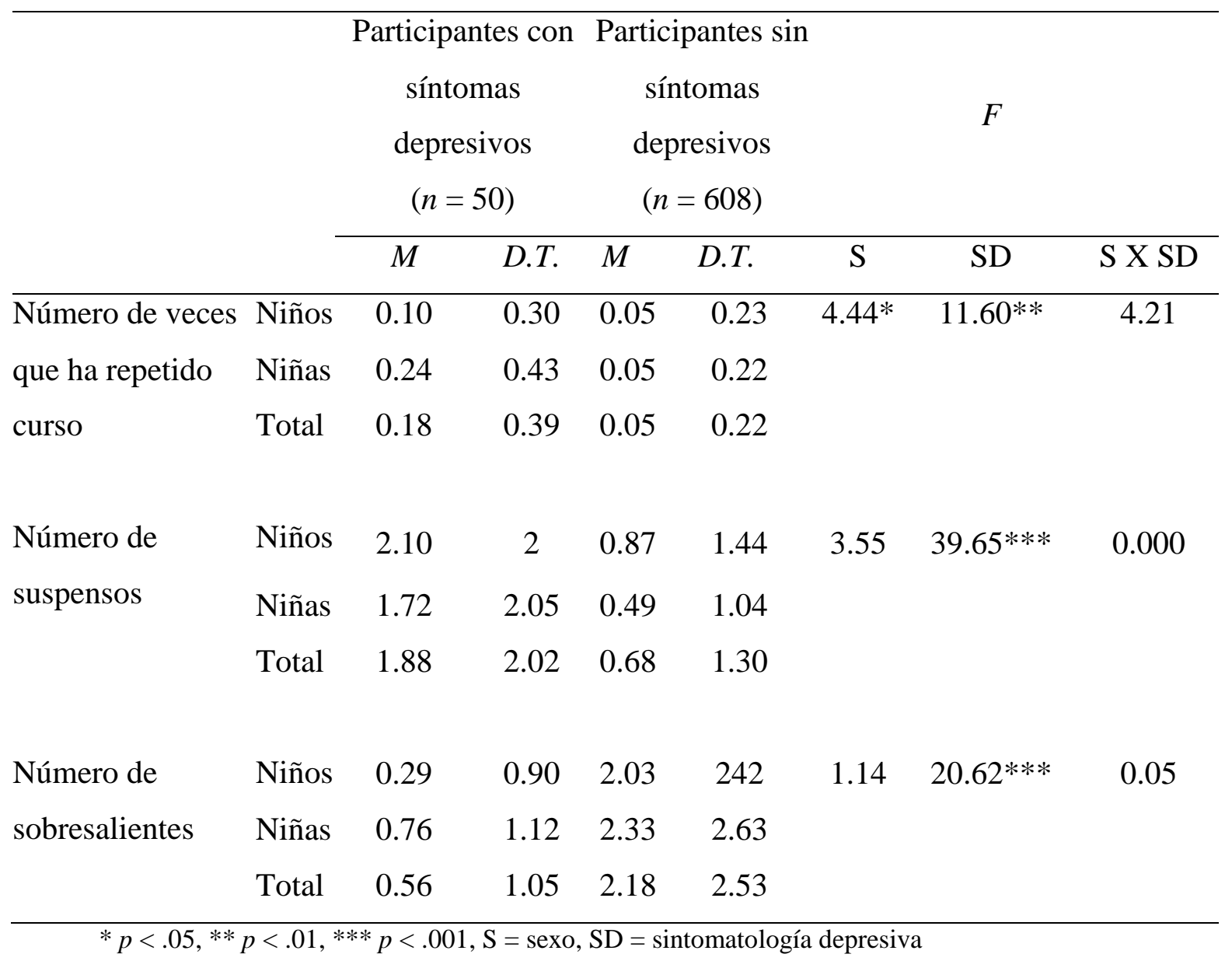

\section{Discusión}

El objetivo de esta investigación era analizar si el rendimiento académico infantil difiere en función de manifestar o no sintomatología depresiva, teniendo en cuenta el sexo y la edad. En concreto se examinó el número de suspensos, el número de sobresalientes y el número de veces que los participantes habían repetido curso. Los resultados obtenidos confirman la existencia de diferencias estadísticamente significativas en el rendimiento escolar, con un mayor número de repeticiones de curso, más suspensos y menos sobresalientes en los niños con síntomas depresivos. La interacción entre manifestar sintomatología depresiva y el sexo no fue significativa para ninguna de las variables estudiadas, y únicamente se hallaron diferencias en el número de cursos repetidos en función del sexo. 
Estudios anteriores que han examinado el rendimiento académico en niños con depresión coinciden con nuestros hallazgos. En uno de los primeros trabajos llevados a cabo sobre esta temática, Bauersfeld (1972) observó que más de un 10\% de los niños que presentaban fracaso escolar manifestaban también depresión. Con población española, Doménech y Polaino-Lorente (1990) encontraron que entre los niños que habían repetido curso alguna vez se daba una mayor incidencia de depresión. Del mismo modo, en un estudio del rendimiento escolar en las asignaturas de lenguaje, matemáticas y sociales se concluyó que el porcentaje de suspensos era mayor cuando los niños tenían sintomatología depresiva (Cabrera y Jiménez, 1990). Con adolescentes el rendimiento escolar también se ha asociado a la depresión. Pérez y Urquijo (2001) examinaron la relación entre la depresión y el rendimiento en las materias de lengua y matemáticas, demostrando que conforme aumentaban los niveles de depresión disminuían las puntuaciones en el desempeño académico de estas materias. En la búsqueda de un modelo predictor de la depresión infantil, Bernaras et al. (2013) estudiaron la relación entre el rendimiento académico entendido como el desempeño del alumno en comparación con el resto de compañeros de clase, encontrando una correlación inversa y significativa entre la sintomatología depresiva y el rendimiento académico.

Investigaciones internacionales también ofrecen resultados consistentes con los obtenidos en el presente estudio. Con adolescentes mexicanos se ha hallado una relación inversa entre el nivel de rendimiento escolar y depresión, de modo que, a mayor sintomatología depresiva, menor rendimiento en matemáticas, inglés y geografía (Galicia, Sánchez y Robles, 2009). Asimismo, Owens, Stevenson, Hadwin, y Norgate (2012) estudiaron la relación entre el afecto negativo, la preocupación, la memoria de trabajo y el rendimiento escolar, concluyendo que los niveles más altos de ansiedad y depresión se asociaban a un menor rendimiento académico. Por otro lado, la sintomatología depresiva se ha considerado como una variable que puede afectar negativamente al rendimiento en las medidas cognitivas y académicas tales como habilidades matemáticas básicas, ortografía, escritura temprana y lectura (Lundy, Silva, Kaemingk, Goodwin y Quan, 2010). Otros estudios han mostrado que los niños con depresión tienen peor desempeño en solución de problemas (Emerson, Mollet y Harrison, 2005) y en memoria de trabajo (Matthews, Coghill, y Rhodes, 2008).

En cuanto a las diferencias en el rendimiento escolar en función del sexo, contrariamente a lo esperado las niñas mostraron un peor rendimiento académico que los niños. En concreto, y aunque el tamaño del efecto es pequeño, repiten más cursos. La mayoría de los 
estudios previos con población general han mostrado un mejor rendimiento en las niñas que en los niños (Echavarri, et al., 2007; Plazas, et al., 2006; Requena, 1998; Sarmiento et al., 2012). Aunque la justificación de esta divergencia respecto a los trabajos anteriores no está clara, es posible que los síntomas depresivos afecten más a las chicas que a los chicos y repercutan por ello en mayor medida en su rendimiento académico. En nuestro estudio, el número de repeticiones de curso es mayor en las niñas con sintomatología depresiva que en los niños, pero no difiere cuando los participantes no manifiestan síntomas depresivos.

El estudio presenta algunas limitaciones que deben tenerse en cuenta para la interpretación de los resultados. En primer lugar se trata de un estudio transversal por lo que sería conveniente llevar a cabo trabajos longitudinales para conocer la evolución del rendimiento académico en los niños que manifiestan sintomatología depresiva. Por otro lado, sería conveniente controlar en investigaciones futuras otras variables que pueden estar implicadas en el rendimiento escolar, como por ejemplo la motivación, el autoconcepto o el apoyo familiar. Las implicaciones educativas de nuestro trabajo son claras. Es conveniente la detección temprana de sintomatología depresiva en el ámbito escolar con el fin de prevenir problemas de rendimiento académico. Del mismo modo, destaca la importancia de examinar la presencia de síntomas depresivos en aquellos escolares cuyos resultados académicos descienden respecto a etapas anteriores, con el objetivo de mejorar su rendimiento escolar en el contexto de la intervención de su problema emocional. 


\section{Referencias}

Bauersfeld, K. H. (1972). Diagnosis and treatment of depressive conditions at a School psychiatric center. Stockholm: Almquist and Wiksell.

Bernaras, E., Jaureguizar, J., Soroa, M., Ibabe, I. y de las Cuevas, C. (2013). Evaluación de la sintomatología depresiva en el contexto escolar y variables asociadas. Anales de Psicología, 29(1), 131-140. doi: 10.6018/analesps.29.1.137831

Blechman, E. A., Mcenroe, M. J., Carella, E. T. y Audette, D. P. (1986). Childhood competence and depression. Journal of Abnormal Psychology, 95, 223-227.

Cabrera, L. y Jiménez, A. B. (1999). Depresión infantil y rendimiento académico: un estudio comparativo entre casos y controles. Revista de Investigación Educativa, 17(1), 89-106.

Cole, D. A. (1991). Preliminary support for a competency-based model of depression in children. Journal of Abnormal Psychology, 100, 181-190. doi: 10.1037/0021-843X.100.2.181

Del Barrio, V. (1997). Depresión infantil. Barcelona: Ariel

Del Barrio, V. (2007). El niño deprimido. Barcelona: Ariel.

Del Barrio, V. y Carrasco, M. A. (2004). CDI: Inventario de Depresión Infantil. Madrid: TEA Ediciones.

Doménech, E. y Polaino-Lorente, A. (1990). Epidemiología de la depresión infantil. Barcelona: Espaxs.

Echavarri, M., Godoy, J. C. y Olaz, F. (2007). Diferencias de género en habilidades cognitivas y rendimiento académico en estudiantes universitarios. Universitas Psychologica, 6(2), 319-329.

Emerson, C. S., Mollet, G. A. y Harrison, D. W. (2005). Anxious-depression in boys: An evaluation of executive functioning. Archives of Clinical Neuropsychology, 20, 539-546. doi: 10.1016/j.acn.2004.10.003

Ezpeleta, L., Osa, N., Gratacos, L. L. y Pons, D. (1992). Depresión y factores asociados en niños con puntuaciones extremas en el CDI. Revista de Psiquiatría Infanto Juvenil, 1, 949.

Feshbach, N. D. y Feshbach, S. (1987). Affective processes and academic achievement. Child Development, 58, 1335-1347. doi: 10.2307/1130625

Fletcher, J. M. (2008). Adolescent depression: diagnosis, treatment and educational attainment. Health Economics, 17(11), 1215-1235. doi: 10.1002/hec.1319 
Galicia, I. X., Sánchez, A. y Robles, F. J. y (2009). Factores asociados a la depresión en adolescentes: Rendimiento escolar y dinámica familiar. Anales de Psicología, 25(2), 227240.

Kaslow, N. J., Rehm, L. P. y Siegel, A. W. (1984). Social-cognitive and cognitive correlates of depression in children. Journal of Abnormal Child Psychology, 12, 605-620.

Kovacs, M. (1992). Manual of the Children's Depression Inventory. Toronto: Multi-Heath Systems.

Levendosky, A. A., Okun, A. y Parker, J. G. (1995). Depression and maltreatment as predictors of social competence and social problem solving skills in school age children. Child Abuse \& Neglect, 19, 1183-1195. doi: 10.1016/0145-2134(95)00086-N

López, C., Alcántara, M. V., Fernández, V., Castro, M. y López, J. A. (2010). Características y prevalencia de los problemas de ansiedad, depresión y quejas somáticas en una muestra clínica infantil de 8-12 años, mediante el CBCL. Anales de Psicología, 26, 325-334.

Lundy, S. M., Silva, G. E., Kaemingk, K. L., Goodwin, J. L. y Quan, S. F. y (2010). Cognitive Functioning and Academic Performance in Elementary School Children with Anxious/Depressed and Withdrawn Symptoms. Open Pediatric Medicine Journal, 4, 1-9. doi: $10.2174 / 1874309901004010001$

Matthews, K., Coghill, D. y Rhodes, S. (2008). Neuropsychological functioning in depressed adolescent girls. Journal of Affective Disorders, 111(1), 113-118. doi: 10.1016/j.jad.2008.02.003

Nissen, G. (1989). Prevención de la depresión infantil en la escuela. Psicopatología, 4(9), 171-176.

Owens, M., Stevenson, J., Hadwin, J. A. y Norgate, R. (2012). Anxiety and depression in academic performance: An exploration of the mediating factors of worry and working memory. School Psychology International, 33(4), 433-449. doi: 10.1177/ 0143034311427433.

Patterson, G. R. y Stoolmiller, M. (1991). Replications of a dual failure model for boys' depressed mood. Journal of Consulting and Clinical Psychology, 59, 491-498. doi: 10.1037/0022-006X.59.4.491

Plazas, A., Aponte, R. y López, S. E. (2006). Relación entre estatus sociométrico, género y rendimiento académico. Psicología desde el Caribe, 17, 176-195.

Requena, F. (1998). Género, redes de amistad y rendimiento académico. Papers, 56, 233-242. 
Roeser, R. W., Van der Wolf, K. y Strobel, K. R. (2001). On the relation between socialemotional and school functioning during early adolescence. Preliminary findings from Dutch and American samples. Journal of School Psychology, 39, 111-139. Doi: http://dx.doi.org/10.1016/S0022-4405(01)00060-7

Sarmiento, C., Vargas, M. y Díaz, R. (2012). Diferencias en el rendimiento escolar por género asociadas al funcionamiento familiar de estudiantes de Bachillerato. Quaderns de Psicología, 14(2), 17-23.

Suhrcke, M. y Nieves, C. (2011). The impact of health and health behaviours on educational outcomes in high-incomes countries: a review of the evidence. Copenhagen: WHO regional Office for Europe. 